\title{
Sonographic diagnosis of partial versus complete molar pregnancy: A reappraisal
}

\author{
Abstract: \\ Objective: To assess the frequency of prospective sonographic diagnosis of molar pregnancy and \\ compare sonographic features of complete vs. partial molar pregnancy.
}

Methods: IRB approved retrospective chart review revealed 70 women with histopathologic diagnosis of molar pregnancy and available ultrasound images from 2001-2011. Clinical data, images, and reports were reviewed, and features enumerated by blinded radiologists.

Results: Mean age was 30.5 years (range 16-49, SD 7.0) with 3.2 (range 1-11, SD 2.3) prior pregnancies. Mean gestational age was 74.0 days (range 39-138, SD 19.1) and serum beta-hCG 131,092 mIU/ml (range 447-662,000, SD 156,191). Pathologic results showed 48 partial and 22 complete molar pregnancies, Sonographically, partial moles more commonly showed a yolk sac ( 56.3 vs. $0 \%, p<.0001)$, fetal pole ( 62.5 vs. $4.6 \%, p<.0001)$, fine septa within the sac ( 25.0 vs. $4.6 \%, p=.05)$, and normal ( 31.3 vs $0 \%, p=.002$ ) or minimally cystic placenta ( 27.1 vs. $4.6 \%, p=0.49$ ), while complete moles had larger gestational sacs (mean 61.9 vs. $44.4 \mathrm{~mm}, \mathrm{p}=.005$ ), were more often sonographically avascular (45.5 vs. $18.8 \%, p=.02$ ), often had abnormal tissue in the uterus ( 82.6 vs. $20.8 \%, p<.0001$ ) and placental masses (86.9 vs. $16.7 \%, p<.0001)$ and were more often prospectively diagnosed $(86.4$ vs. $41.7 \%, p=.0005)$. Conclusion: Complete molar pregnancy is associated with marked cystic change and mass formation, and is often diagnosed sonographically. Partial molar pregnancy often presents with minor cystic changes of placenta and remains underdiagnosed sonographically. However, correct prospective diagnosis was more frequent than in older reports, perhaps due to improved spatial resolution of ultrasound.

Key words: molar pregnancy, hydatidiform mole, gestational trophoblastic disease , ultrasound, obstetrics

This is the author manuscript accepted for publication and has undergone full peer review but has not been through the copyediting, typesetting, pagination and proofreading process, which may lead to differences between this version and the Version record. Please cite this article as doi:10.1002/ jcu.22410. 


\section{INTRODUCTION}

About in 1 in 700 pregnancies in the United States is complicated by partial hydatidiform mole, while complete hydatidiform moles occur in 1 in 1500 pregnancies ${ }^{1}$. Molar pregnancies are non-viable gestations by definition, with tissue types varying according to their chromosomal makeup. Partial molar pregnancies are formed by an abnormal combination of an ovum and one or more spermatozoa, containing a range of fetal parts in combination with abnormally proliferative chorionic villi. Complete moles are entirely paternal in origin, arising from one or more spermatozoa combining with a non-viable ovum with no normal fetal tissue ${ }^{2}$. Approximately $10-20 \%$ of women with complete molar pregnancies and $0.5 \%-11 \%$ of partial molar pregnancies will go on to develop persistent, invasive gestational trophoblastic disease including invasive mole, choriocarcinoma, and placental type trophoblastic tumor ${ }^{1,3}$. Clinical suspicion of hydatidiform mole in failed pregnancy has several potential impacts on clinical management, including determination of need and type of uterine evacuation, submission of products of conception to pathology, and serum ?eta-human chorionic gonadotropin (beta-hCG) surveillance ${ }^{4}$. Obstetric ultrasound practitioners, including radiologists, have the potential to significantly impact patient care by prospectively diagnosing molar pregnancy.

The typically described ultrasound appearance of complete molar pregnancy includes a complex, multicystic, and often hypervascular intrauterine mass, and an absence of fetal tissue ${ }^{5-7}$. Partial molar pregnancy may present as a subtle placental abnormality with a live embryo, a spontaneous intrauterine demise, or an empty gestational sac ${ }^{5}$. Criteria for specific ultrasound findings in partial molar pregnancy have been suggested in the literature, including transverse : anteroposterior gestational sac diameter ratio $>1.5$, and cystic change or irregularity of the decidua, placenta, or myometrium $^{5,8}$. 
In the first trimester, frequency of sonographic diagnosis of complete mole is higher than that of partial mole, and improves with increasing GA. Fowler et al. reviewed imaging from 378 pathology proven molar pregnancies, demonstrating pre-evacuation ultrasound diagnosis in 200 out of 253 (79\%) of complete hydatidiform moles and 178 out of 616 (29\%)of partial hydatidiform moles, with a trend toward improved diagnostic accuracy with increasing GA ${ }^{1}$. Other authors have demonstrated similar trends $s^{5,9}$.

We hypothesize that despite development of more sensitive beta-hCG serum assays and widespread use of pelvic ultrasound in the decade that followed these studies, molar pregnancy remains prospectively underdiagnosed in the first trimester. The aim of our study is to examine the sensitivity of contemporary first trimester ultrasound for diagnosis of molar pregnancy, and to describe sonographic features of pathologically proven complete and partial molar pregnancy.

\section{METHODS}

Institutional review board approval was obtained for a retrospective review of medical records between Jan 1, 2001 to December 31, 2011, revealing 130 women with early pregnancy loss and subsequent diagnosis of molar pregnancy diagnosed during routine histopathologic examination of gestational products. Of these, 70 had ultrasound images available in the radiology archive and were included in this study.

Clinical data including patient age, gravidity and parity, quantitative beta-hCG levels, gestational age (GA) by last menstrual period (LMP), and histopathologic diagnosis were obtained from the medical records. Ultrasound imaging was performed on a GE Logiq 9, GE Logiq E9, or Philips iU22 and included transabdominal (3-8 MHz) and transvaginal (5-10 MHz) pelvic imaging with grey scale and cine-capture series on all patients. Color and spectral Doppler images were obtained when desired by the sonographer and/or interpreting sonologist in order to further characterize the findings on greyscale 
images. All original images, including cine series, were available for retrospective review using a Syngo Dynamics ultrasound workstation (Siemens Healthcare USA). Images were reviewed in consensus by two abdominal imaging fellowship-trained radiologists with 5 and 8 years of subspecialty experience respectively, blinded to the specific type of molar pregnancy. Data regarding the presence of a gestational sac, minimum sac diameter, maximum sac diameter, ratio of maximum:minimum sac diameter, mean sac diameter, presence of septa within the gestational sac, presence of a fetal pole and fetal heart rate, sonographic GA, yolk sac, abnormal tissue, vascularity of abnormal tissue, and placental abnormalities were recorded. A gestational sac was considered to be present when an anechoic or hypoechoic collection was noted within the canal and measured by the sonographer. Sonographic GA was assessed on the basis of fetal pole when present or best assessment of mean gestational sac diameter otherwise. Abnormal tissue was defined as the presence of non-fluid material in the endometrial canal that could not be clearly identified as normal gestational products. Because color Doppler images were not performed with standardized settings, the myometrium was used as an internal control for evaluation of vascularity of endometrial contents. The vascularity of gestational products was scored on a 0-2 scale (Figure 1), where $0=$ no demonstrable vascularity, 1=similar vascularity to myometrium, and $2=$ greater vascularity than myometrium. The placenta was rated 0-3

(Figure 2) where $0=$ sonographically normal, 1=minor cystic changes, $2=$ substantially cystic placenta, and 3=mass in place of normal lentiform placenta. The original study dictations were reviewed to determine whether the interpreting radiologist made the prospective diagnosis of molar pregnancy. The prospective diagnosis was considered positive if the original dictation described a possible or probable diagnosis of molar pregnancy. The specific subtype of molar pregnancy was rarely commented upon in the original dictations and was not quantified.

Descriptive statistics were performed using means, counts and percentages. Comparisons were made between the partial and complete molar pregnancy pathologic groups using t-tests for continuous 
variables and chi-square test or Fisher's exact test for categorical variables. The placenta and vascularity rating scales were analyzed both as continuous and as categorical variables. Multivariate logistic regression analysis of factors contributing to correct prospective diagnosis was performed using stepwise forward selection of variables, initially including all clinically relevant contributing features: age; parity; serum beta-hCG; GA by sonography and LMP; sac diameters; presence of yolk sac, fetal pole, septa and/or abnormal tissue; placental and vascularity ratings. $\mathrm{P}<.05$ was the standard for inclusion in logistic regression and overall statistical significance. All analyses were performed using SAS 9.3, SAS Institute, Cary, NC.

\section{RESULTS}

The mean age of women in the study population was 30.5 years (range 16-49, SD 7.0) and mean number of pregnancies including the current gestation was 3.2 (range 1-11, SD 2.3). The mean GA by last menstrual period (LMP) was 74.0 days (range 39-138, SD 19.1) with serum beta-hCG 131,092 $\mathrm{mIU} / \mathrm{ml}$ (range 447-662,000, SD 156,191) within a day of ultrasound imaging. 48 women $(68.6 \%)$ had partial molar pregnancies and 22 women (31.4\%) had complete molar pregnancies after histopathologic examination of uterine contents. The demographic and clinical information in each group is provided in Table 1.

Specific sonographic features and frequency of prospective diagnosis of molar pregnancy in the original dictation are enumerated, and compared between partial and complete molar pregnancy groups, in Table 2. Complete molar pregnancies had significantly larger gestational sacs, more frequent findings of abnormal tissue, and greater abnormalities of the placenta. In fact, 19 (86.4\%) of the complete molar pregnancies had frankly masslike placentas compared with only $8(16.7 \%)$ of the partial molar pregnancies $(\mathrm{p}<.0001)$. Partial molar pregnancies more frequently demonstrated distinct yolk sacs and fetal poles and tended to be more vascular. Nine (18.8\%) partial and 10 (45.5\%) complete 
moles were avascular by color and/or power Doppler $(\mathrm{p}=.02)$. Thin septa within the gestational sac were more frequently observed in partial molar pregnancies (Figure 3).

In multivariate logistic regression, GA by LMP, presence of a yolk sac, and placental ratings were retained as statistically significant predictors of correct prospective sonographic diagnosis of molar pregnancy. The overall score test for the model indicated a superior fit to the null model $(p<.0001)$. For every 1 point increase in placenta score, there was a $>999$-fold increased odds of correct prospective diagnosis $(95 \% \mathrm{Cl} 1.13,>999.99, \mathrm{p}=.04)$. For every 1 day increase in GA by $L M P$, the odds of correct prospective diagnosis increased 1.19 -fold $(95 \% \mathrm{Cl} 0.97,1.47, \mathrm{p}=0.10)$. The presence of a yolk sac increased the odds of correct prospective diagnosis by 514.66 -fold $(95 \% \mathrm{Cl} 0.19,>999.99, \mathrm{p}=.12$ ).

\section{DISCUSSION}

Complete molar pregnancy has characteristic and often strikingly abnormal sonographic features, while partial molar pregnancy may exhibit only subtle abnormalities ${ }^{10}$. As such, complete molar pregnancy was diagnosed prospectively by ultrasound much more commonly than partial molar pregnancy in this study $(86.4 \%$ vs. $41.4 \%, \mathrm{p}=.0005)$. Detection rates in complete molar pregnancy are reported at $58-95 \%$, and partial molar pregnancy at $17-29 \%^{1,5,9}$. Although partial molar pregnancy remains prospectively underdiagnosed, it appears that there is some improvement in sensitivity compared with these older reports. We suggest that this may be attributed to greater spatial resolution of ultrasound enabling detection of placental cystic changes and/or greater awareness of the diagnosis of molar pregnancy on the part of interpreting sonologists

In multivariate regression, the presence of cystic change in the placenta was a strong predictor of correct sonographic diagnosis, suggesting that radiologists are aware of the importance of this finding and indirectly supporting the idea that improved spatial resolution is important to the trend of improved diagnosis. Both GA by LMP and the presence of a yolk sac were also retained as predictors in 
multivariate analysis, probably both reflections of the same concept: it is easier to identify the changes of molar pregnancy when the gestation is more advanced, the villi more hydropic, and the tissue mass larger $^{11}$. We suspected that radiologist awareness of serum beta-hCG values in the current era of electronic medical records could be a factor in improved prospective diagnosis of molar pregnancy, but the serum beta-hCG levels did not significantly predict correct diagnosis and we therefore did not formally evaluate whether readers were aware of these values at the time of dictation.

Concordant with prior studies ${ }^{1,3,6,12}$, the majority of complete hydatidiform molar pregnancies in this study exhibited exuberant cystic and/or masslike proliferation of placental tissue. The gestational sacs were larger and abnormal tissue was present in most cases. As expected, fetal parts were not identified in complete molar pregnancies, because complete molar pregnancies have no maternal genetic contribution. The study radiologists identified what they believed to be a probable fetal pole only in a single case of complete molar pregnancy.

The range of appearances of partial molar pregnancy was much broader, occupying a spectrum from a tiny empty sac to a 7 week fetal pole with a cystic placenta. 28 (58.33\%) of partial molar pregnancies had a normal placenta or only minor cystic change. We identified a particular sonographic appearance of multiple thin septa within the gestational sac in a subset of cases, which was more common in partial molar pregnancies $(25.0 \%$ vs. $4.6 \%, p=.05)$. Although insensitive, this sign may favor diagnosis of molar pregnancy in a first trimester gestation. However, the positive predictive value of this finding remains incompletely assessed without a set of non-molar controls. Partial moles were more vascular than complete moles, although in contrast to prior reports hypervascularity was uncommon in both types of molar pregnancy ${ }^{13}$. The majority of both partial and complete molar pregnancies had sac size ratios $>1.5$, but the mean values did not differ significantly between groups. 
Our study is limited by its retrospective nature and sample size. Ultrasound machines and imaging techniques varied over the years when these women were scanned, and there is inherent heterogeneity of the images in this regard. In particular, Doppler imaging was employed in an ad hoc fashion and technical parameters including transducer frequency, angle of insonation, gain, and pulse repetition frequency in color Doppler and the variable use of power Doppler could not be controlled. Second, we used the originally dictated reports as the outcome variable for assessing prospective diagnosis, though not for evaluation of specific imaging features. Using the originally dictated report allows us to report on real life clinical practice but also introduces unquantified variation among readers. Finally, we are unable to comment on true diagnostic specificity and positive predictive value of ultrasound for detection of molar pregnancy in the absence of a set of non-molar pregnancy controls.

In conclusion, complete molar pregnancies are often correctly diagnosed sonographically, and discrete placental abnormalities along a spectrum from cystic change to overt masses are apparent in most cases. Partial molar pregnancy often presents with a recognizable yolk sac and sometimes a fetal pole (rarely with fetal cardiac motion), in the setting of mild to moderate cystic changes in placenta, and remains underdiagnosed prospectively. However, there is an increase in diagnostic sensitivity compared to older studies, perhaps owing to improved spatial resolution of ultrasound enabling detection of subtle cystic placental change and/or increased physician awareness of this diagnosis. Given the potential impact on clinical management, interpreting sonologists should remain alert to the possibility of molar pregnancy in first trimester pregnancy loss. 


\section{References}

1. Fowler DJ, Lindsay I, Seckl MJ, et al. Routine pre-evacuation ultrasound diagnosis of hydatidiform mole: experience of more than 1000 cases from a regional referral center. Ultrasound in obstetrics \& gynecology : the official journal of the International Society of Ultrasound in Obstetrics and Gynecology 2006;27:56.

2. Sun SY, Melamed A, Goldstein DP, et al. Changing presentation of complete hydatidiform mole at the New England Trophoblastic Disease Center over the past three decades: Does early diagnosis alter risk for gestational trophoblastic neoplasia? Gynecologic oncology 2015;138:46.

3. Johns J, Greenwold N, Buckley S, et al. A prospective study of ultrasound screening for molar pregnancies in missed miscarriages. Ultrasound in obstetrics \& gynecology : the official journal of the International Society of Ultrasound in Obstetrics and Gynecology 2005;25:493.

4. Berkowitz RS, Goldstein DP. Current management of gestational trophoblastic diseases. Gynecologic oncology 2009;112:654.

5. Kirk E, Papageorghiou AT, Condous G, et al. The accuracy of first trimester ultrasound in the diagnosis of hydatidiform mole. Ultrasound in obstetrics \& gynecology : the official journal of the International Society of Ultrasound in Obstetrics and Gynecology 2007;29:70.

6. Benson CB, Genest DR, Bernstein MR, et al. Sonographic appearance of first trimester complete hydatidiform moles. Ultrasound in obstetrics \& gynecology : the official journal of the International Society of Ultrasound in Obstetrics and Gynecology 2000;16:188.

7. Fine C, Bundy AL, Berkowitz RS, et al. Sonographic diagnosis of partial hydatidiform mole. Obstetrics and gynecology 1989;73:414.

8. Jauniaux E. Ultrasound diagnosis and follow-up of gestational trophoblastic disease. Ultrasound in obstetrics \& gynecology : the official journal of the International Society of Ultrasound in Obstetrics and Gynecology 1998;11:367.

9. Sebire NJ, Rees H, Paradinas F, et al. The diagnostic implications of routine ultrasound examination in histologically confirmed early molar pregnancies. Ultrasound in obstetrics \& gynecology : the official journal of the International Society of Ultrasound in Obstetrics and Gynecology 2001;18:662.

10. Shanbhogue AK, Lalwani N, Menias CO. Gestational trophoblastic disease. Radiologic clinics of North America 2013;51:1023.

11. Fowler DJ, Lindsay I, Seckl MJ, et al. Histomorphometric features of hydatidiform moles in early pregnancy: relationship to detectability by ultrasound examination. Ultrasound in obstetrics \& gynecology : the official journal of the International Society of Ultrasound in Obstetrics and Gynecology 2007;29:76.

12. Lazarus E, Hulka C, Siewert B, et al. Sonographic appearance of early complete molar pregnancies. Journal of ultrasound in medicine : official journal of the American Institute of Ultrasound in Medicine 1999;18:589.

13. Zhou Q, Lei $X Y, X i e ~ Q$, et al. Sonographic and Doppler imaging in the diagnosis and treatment of gestational trophoblastic disease: a 12-year experience. Journal of ultrasound in medicine : official journal of the American Institute of Ultrasound in Medicine 2005;24:15. 
Figure legends

Figure 1: Vascularity rating scale illustrated by transvaginal ultrasound in three women with molar pregnancies. A) Vascularity $=0$. No demonstrable color Doppler vascularity in hydropic villi (arrow) in an 18 year old woman with complete mole. B) Vascularity =1. Power Doppler flow within placental issue (arrow) is similar to the surrounding myometrium in a 20 year old woman with complete mole. C)

Vascularity=2. Focal marked hypervascularity of placenta (arrow) by power Doppler in a 40 year old woman with partial mole.

Figure 2: Placental rating scale illustrated in four women with molar pregnancies. A) Placenta=1. Normal placenta (arrow) in a 29 year old woman with partial mole. B) Placenta=2. Minor cystic change (arrow) in a 21 year old woman with partial mole. C) Placenta $=3$. Substantial cystic change (arrow) in a 28 year old with partial mole. D) Placenta $=4$. Placental mass (arrows) in a 20 year old woman with complete mole.

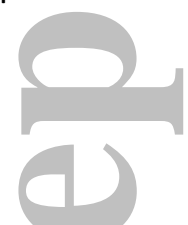

Figure 3: Septa in gestational sac illustrated in two women with molar pregnancies. A) Fine septa (arrow) within the gestational sac in a 37 year old woman with partial mole. B) Slightly thicker septa (arrow) within the gestational sac in a 23 year old woman with partial mole. 


\begin{tabular}{|l|c|c|c|}
\hline \multicolumn{1}{|c|}{ Parameter } & $\begin{array}{c}\text { Partial molar pregnancy } \\
(\mathbf{n = 4 8 )}\end{array}$ & $\begin{array}{c}\text { Complete molar pregnancy } \\
(\mathbf{n = 2 2})\end{array}$ & $\begin{array}{c}\text { P } \\
\text { value }\end{array}$ \\
\hline Age in years & $31.5 \pm 5.8$ & $28.4 \pm 9.1$ & .08 \\
\hline Gravidity & $3.3 \pm 2.2$ & $3.1 \pm 2.5$ & .79 \\
\hline Parity & $1.2 \pm 1.4$ & $1.3 \pm 1.7$ & .73 \\
\hline GA by LMP in days & $76.1 \pm 16.7$ & $69.6 \pm 23.1$ & .19 \\
\hline $\begin{array}{l}\text { Quantitative serum beta-hCG in } \\
\text { mlU/mL }\end{array}$ & $115,952 \pm 135,018$ & $162,031 \pm 187,950$ & .27 \\
\hline
\end{tabular}

Table 1: Clinical and demographic information for 70 women with molar pregnancy. Data are given as mean \pm standard deviation (SD). GA=gestational age, LMP = last menstrual period. 


\begin{tabular}{|c|c|c|c|}
\hline Parameter & $\begin{array}{l}\text { Partial molar pregnancy } \\
\qquad(n=48)\end{array}$ & $\begin{array}{l}\text { Complete molar pregnancy } \\
\qquad(\mathrm{n}=22)\end{array}$ & $\begin{array}{c}P \\
\text { value }\end{array}$ \\
\hline Mean sac diameter in $\mathrm{mm}$ & $33.9 \pm 13.5$ & $49.8 \pm 26.8$ & .001 \\
\hline Maximum sac diameter in $\mathrm{mm}$ & $44.8 \pm 18.2$ & $61.9 \pm 30.8$ & .005 \\
\hline Sac diameter ratio & $2.3 \pm 1.1$ & $1.9 \pm 0.5$ & .10 \\
\hline Yolk sac visualized & 27 (56.3\%) & $0(0 \%)$ & $<.0001$ \\
\hline Fetal pole visualized & $30(62.5 \%)$ & $1(4.6 \%)$ & $<.0001$ \\
\hline Fetal cardiac activity & $1(2.1 \%)$ & $0(0 \%)$ & $.49 *$ \\
\hline Sonographic GA in days & $57.3 \pm 24.8$ & $73.6 \pm 56.3$ & .09 \\
\hline Abnormal tissue noted & $11(22.9 \%)$ & $19(86.4 \%)$ & $<.0001$ \\
\hline Septations within gestational sac & $12(25.0 \%)$ & $1(4.6 \%)$ & $.05 *$ \\
\hline Placenta rating (0-3 scale) & $1.3 \pm 1.1$ & $2.8 \pm 0.5$ & $<.0001$ \\
\hline - $0=$ Normal & $15(31.3 \%)$ & $0(0.0 \%)$ & $.002 *$ \\
\hline - 1 = Minor cystic changes & $13(27.1 \%)$ & $1(4.6 \%)$ & $.049 *$ \\
\hline - 2 = Major cystic changes & $12(25.0 \%)$ & $2(9.1 \%)$ & $.19 *$ \\
\hline - 3 = Placental mass & $8(16.7 \%)$ & 19 (86.4\%) & $<.0001$ \\
\hline Vascularity rating (0-2 scale) & $1.0 \pm 0.6$ & $0.6 \pm 0.6$ & .01 \\
\hline - $0=$ Avascular & $9(18.8 \%)$ & $10(45.5 \%)$ & .02 \\
\hline $\begin{array}{l}\text { - } 1 \text { = Similar vascularity to } \\
\text { myometrium }\end{array}$ & $29(60.4 \%)$ & $10(45.5 \%)$ & .24 \\
\hline $\begin{array}{l}2=\text { Hypervascular to } \\
\text { myometrium }\end{array}$ & $8(16.7 \%)$ & $1(4.5 \%)$ & $.25^{*}$ \\
\hline $\begin{array}{l}\text { Called a possible or probable } \\
\text { molar pregnancy in original } \\
\text { dictation? }\end{array}$ & $20(41.7 \%)$ & 19 (86.4\%) & .0005 \\
\hline
\end{tabular}

Table 2: Sonographic features in 70 molar pregnancies. GA=gestational age. Continuous variables are given as mean \pm standard deviation (SD) and compared using t-tests, and categorical variables as $\mathrm{n}(\%)$ and compared using chi-square test or Fisher's exact test, indicated by *, where cell counts were too low. Statistically significant results $(p<.05)$ are bolded. 


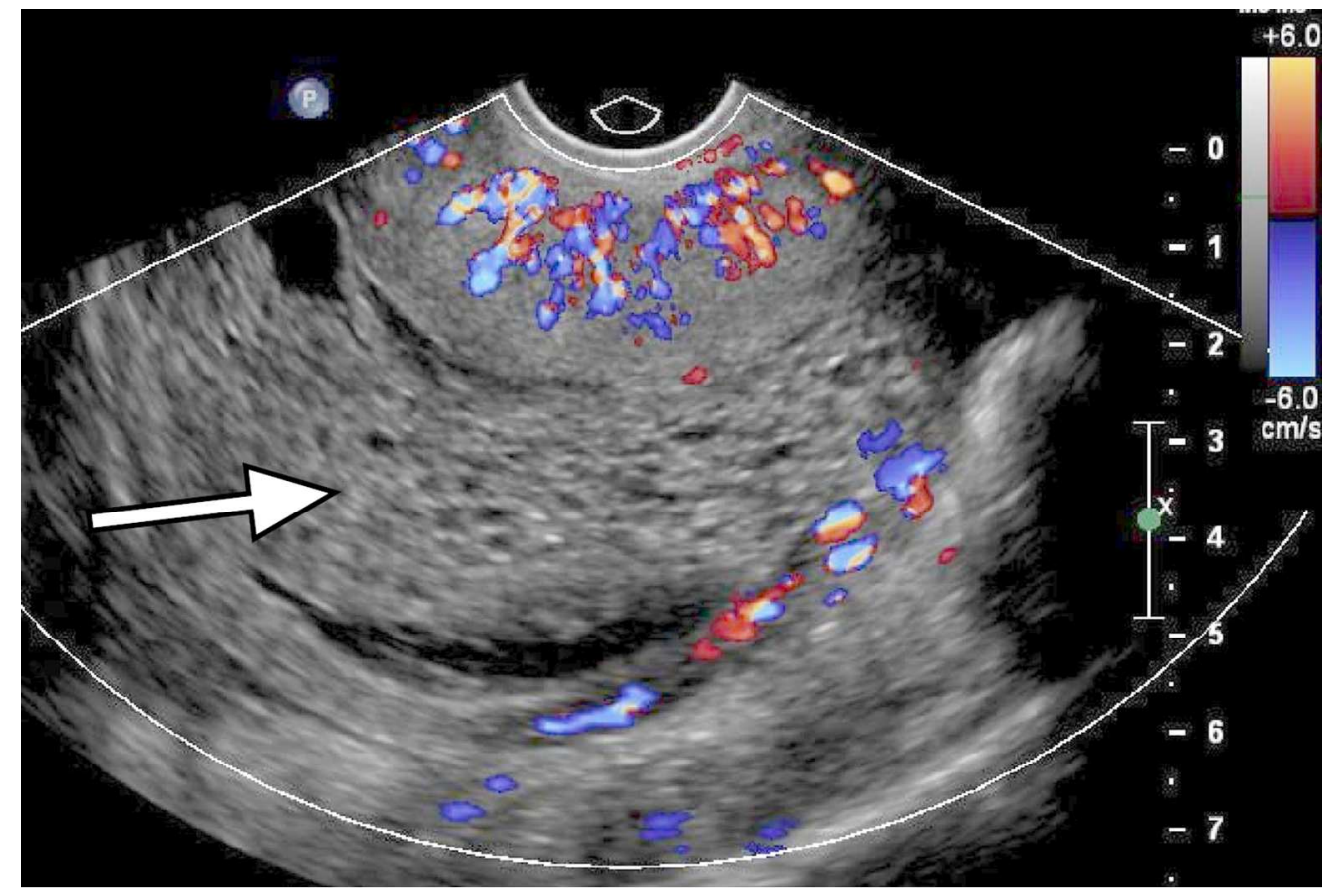

(Figure 1A) Figure 1: Vascularity rating scale illustrated by transvaginal ultrasound in three women with molar pregnancies. A) Vascularity $=0$. No demonstrable color Doppler vascularity in hydropic villi (arrow) in an 18 year old woman with complete mole.

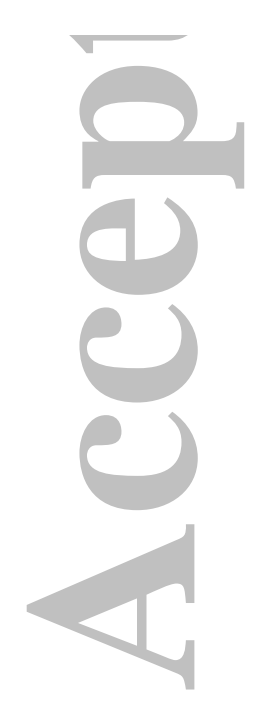

$127 \times 85 \mathrm{~mm}(300 \times 300 \mathrm{DPI})$ 


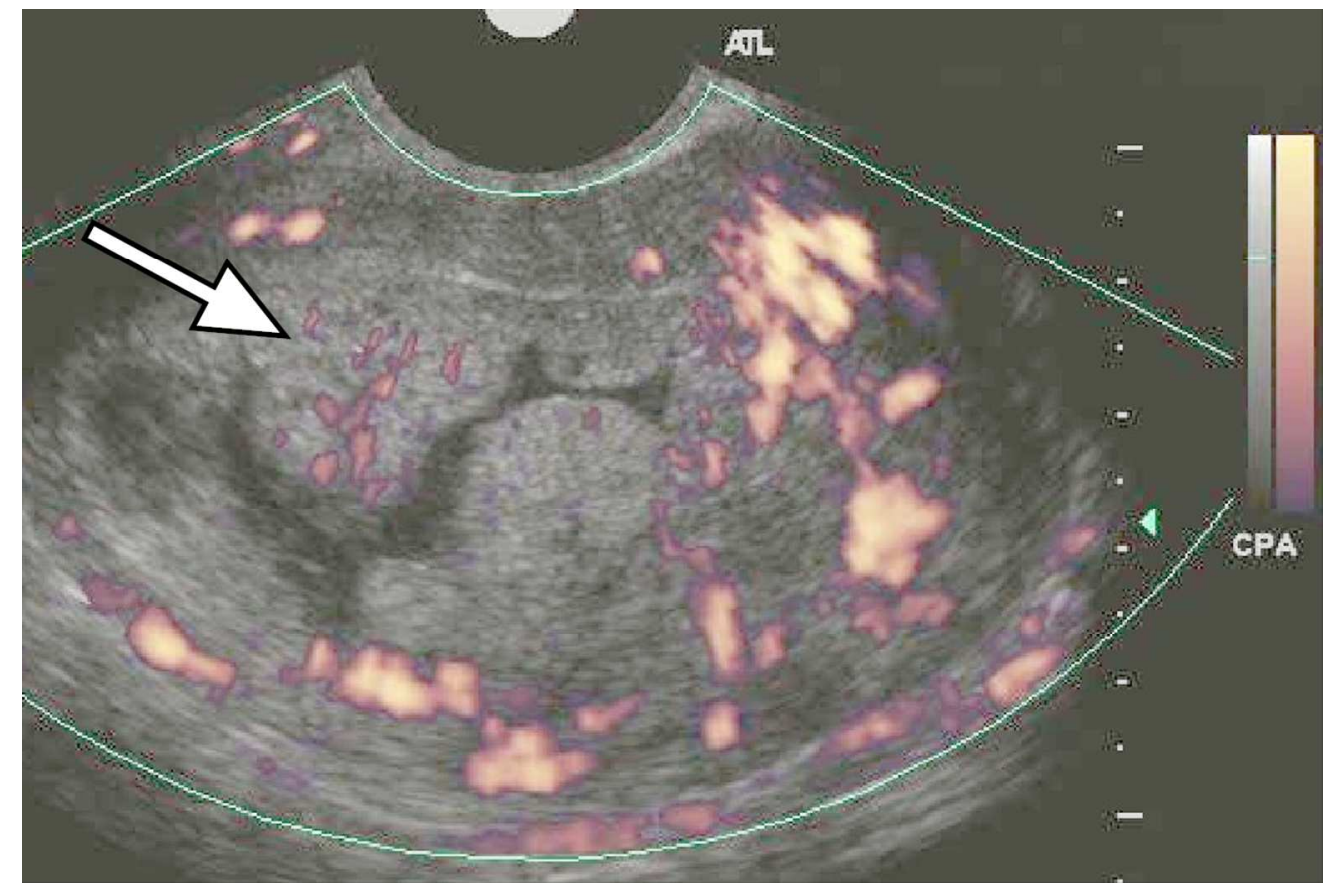

(Figure 1B) Figure 1: Vascularity rating scale illustrated by transvaginal ultrasound in three women with molar pregnancies. B) Vascularity $=1$. Power Doppler flow within placental issue (arrow) is similar to the surrounding myometrium in a 20 year old woman with complete mole.

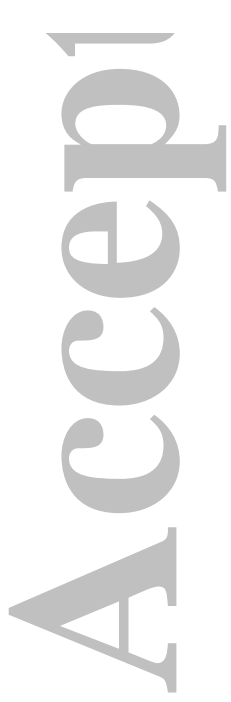

$127 \times 85 \mathrm{~mm}(300 \times 300 \mathrm{DPI})$ 


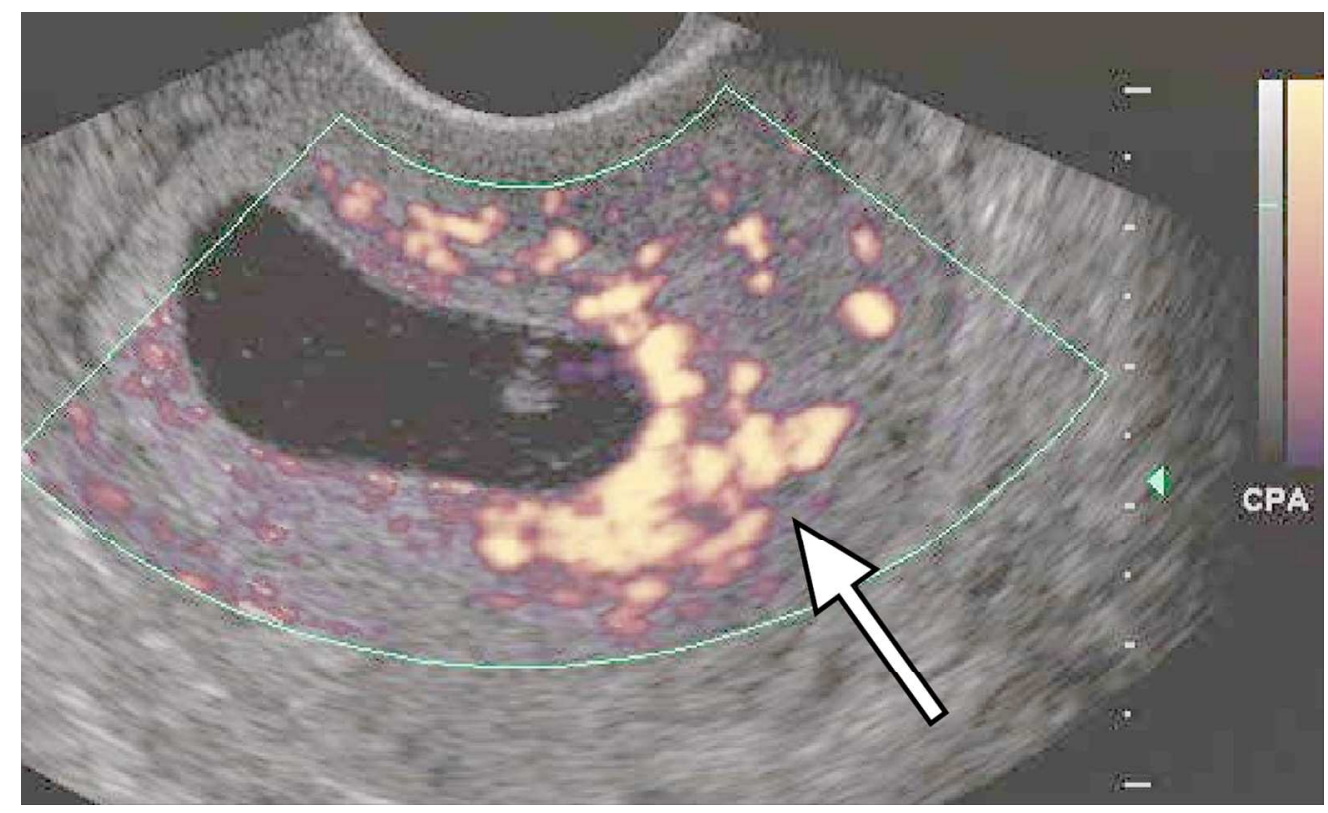

(Figure 1C) Figure 1: Vascularity rating scale illustrated by transvaginal ultrasound in three women with molar pregnancies. C) Vascularity $=2$. Focal marked hypervascularity of placenta (arrow) by power Doppler in a 40 year old woman with partial mole.

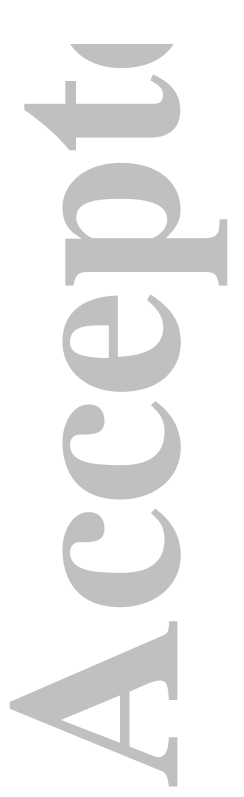

$127 \times 77 \mathrm{~mm}(300 \times 300 \mathrm{DPI})$ 


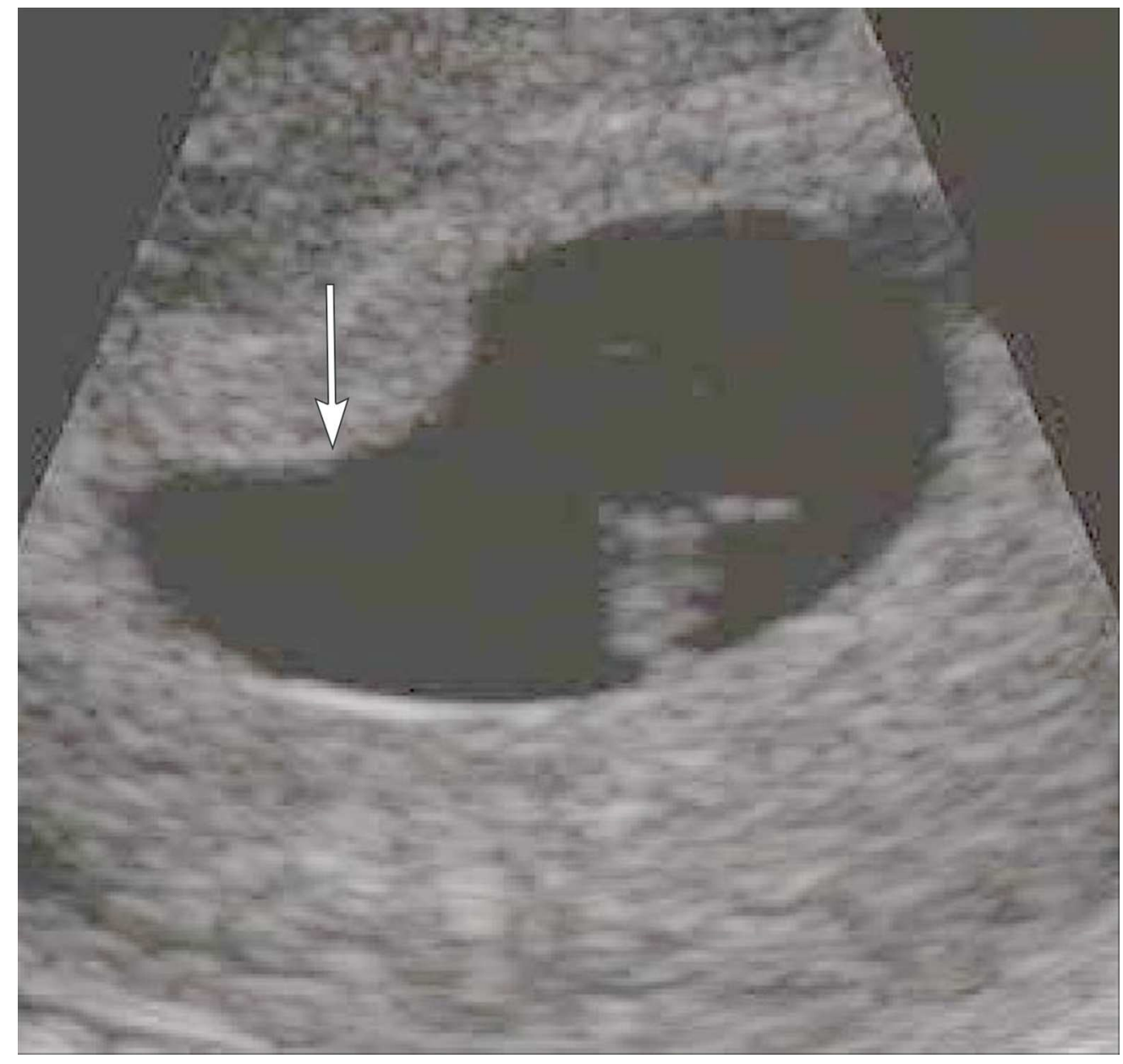

(Figure 2A) Figure 2: Placental rating scale illustrated in four women with molar pregnancies. A) Placenta $=1$. Normal placenta (arrow) in a 29 year old woman with partial mole.

$127 \times 120 \mathrm{~mm}(300 \times 300$ DPI $)$

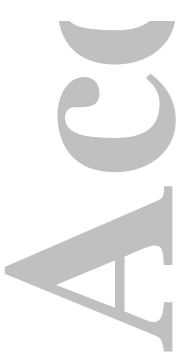

John Wiley \& Sons

This article is protected by copyright. All rights reserved. 


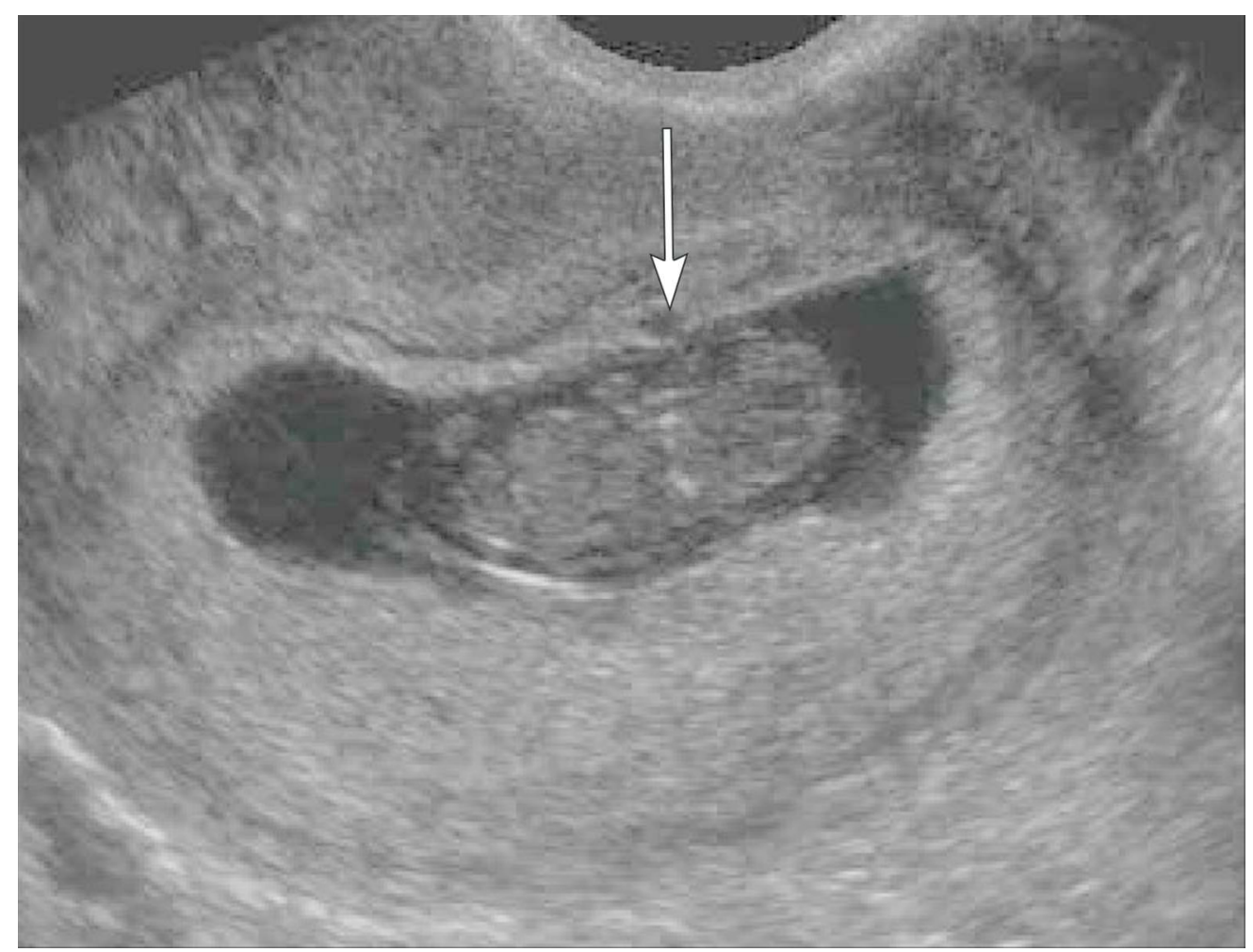

(Figure 2B) Figure 2: Placental rating scale illustrated in four women with molar pregnancies. B) Placenta=2. Minor cystic change (arrow) in a 21 year old woman with partial mole.

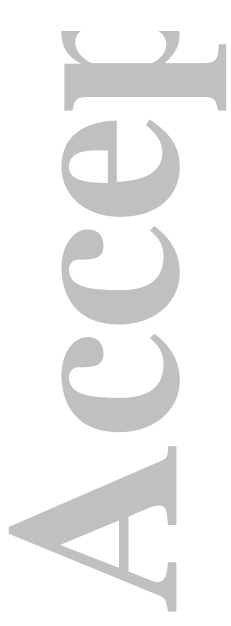

$127 \times 96 \mathrm{~mm}(300 \times 300 \mathrm{DPI})$ 


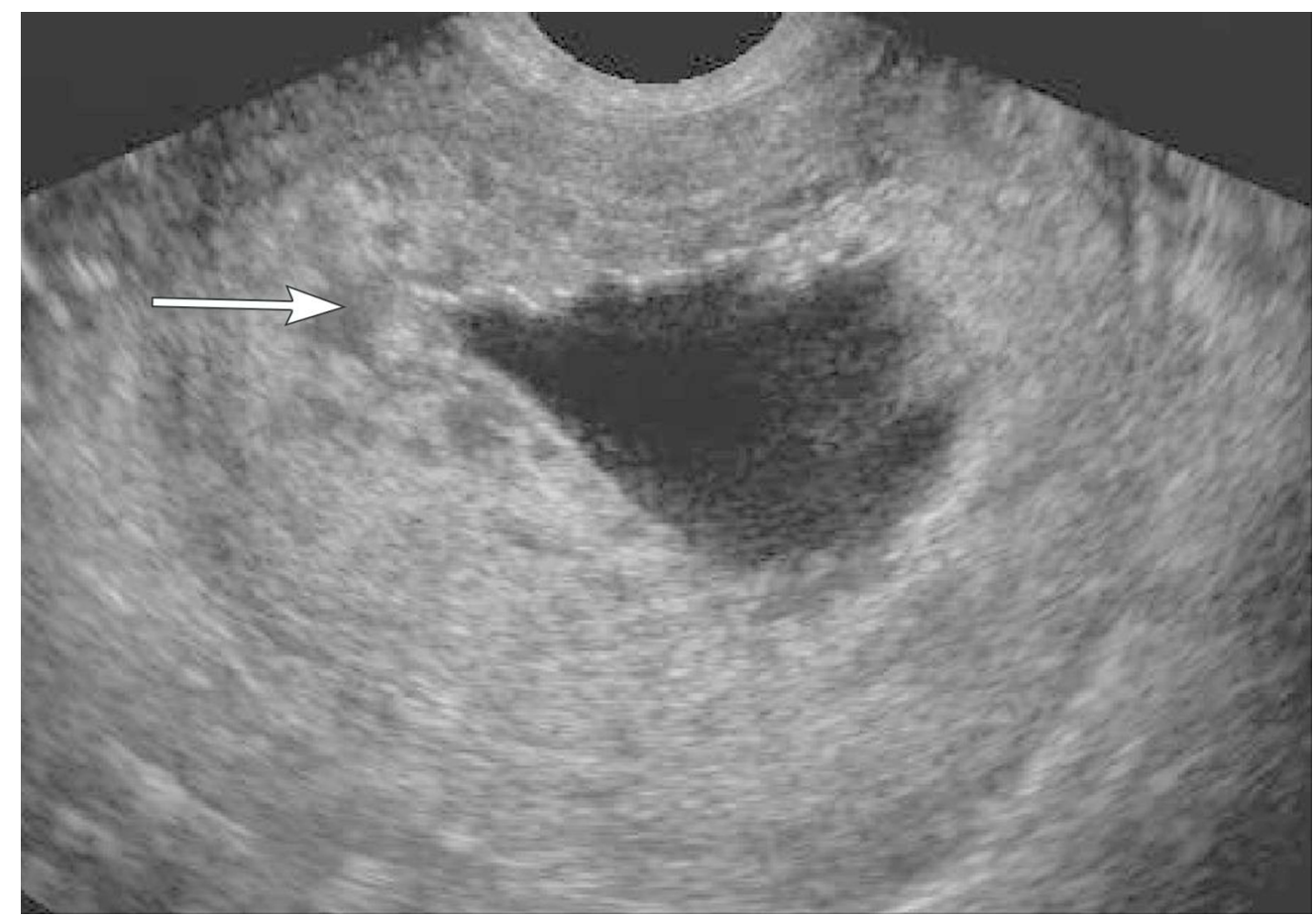

(Figure 2C) Figure 2: Placental rating scale illustrated in four women with molar pregnancies. C) Placenta = 3. Substantial cystic change (arrow) in a 28 year old with partial mole.

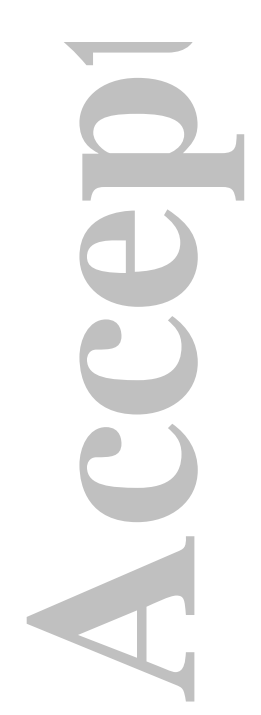

$127 \times 88 \mathrm{~mm}(300 \times 300$ DPI $)$ 


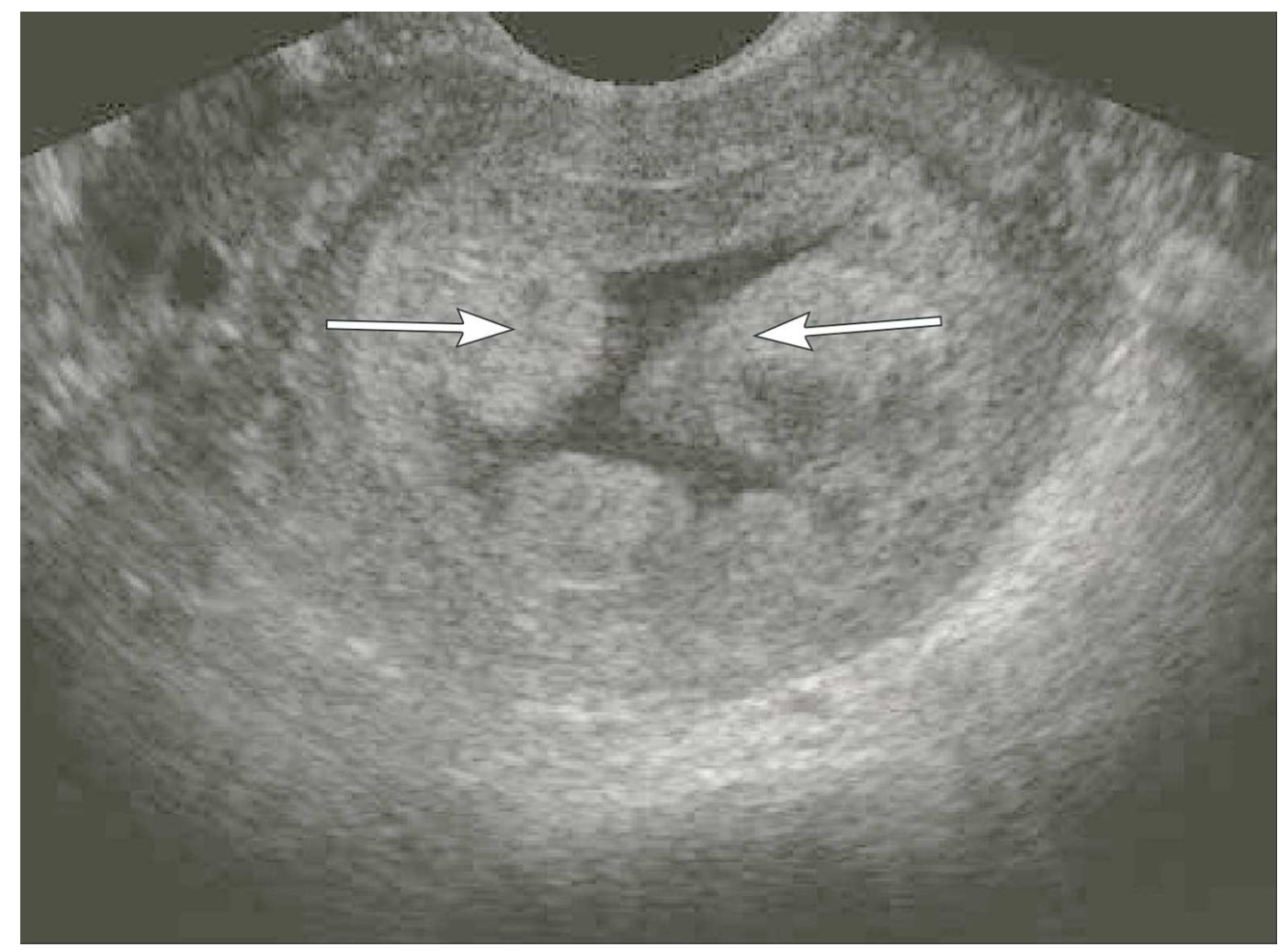

(Figure 2D) Figure 2: Placental rating scale illustrated in four women with molar pregnancies. D) Placenta $=4$. Placental mass (arrows) in a 20 year old woman with complete mole.

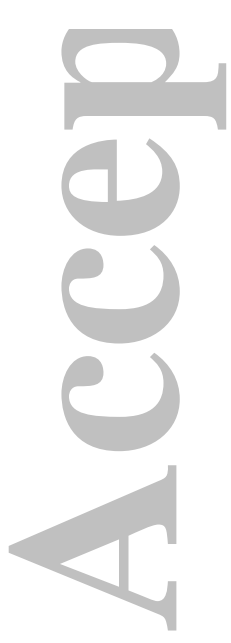

$127 \times 94 \mathrm{~mm}(300 \times 300 \mathrm{DPI})$ 


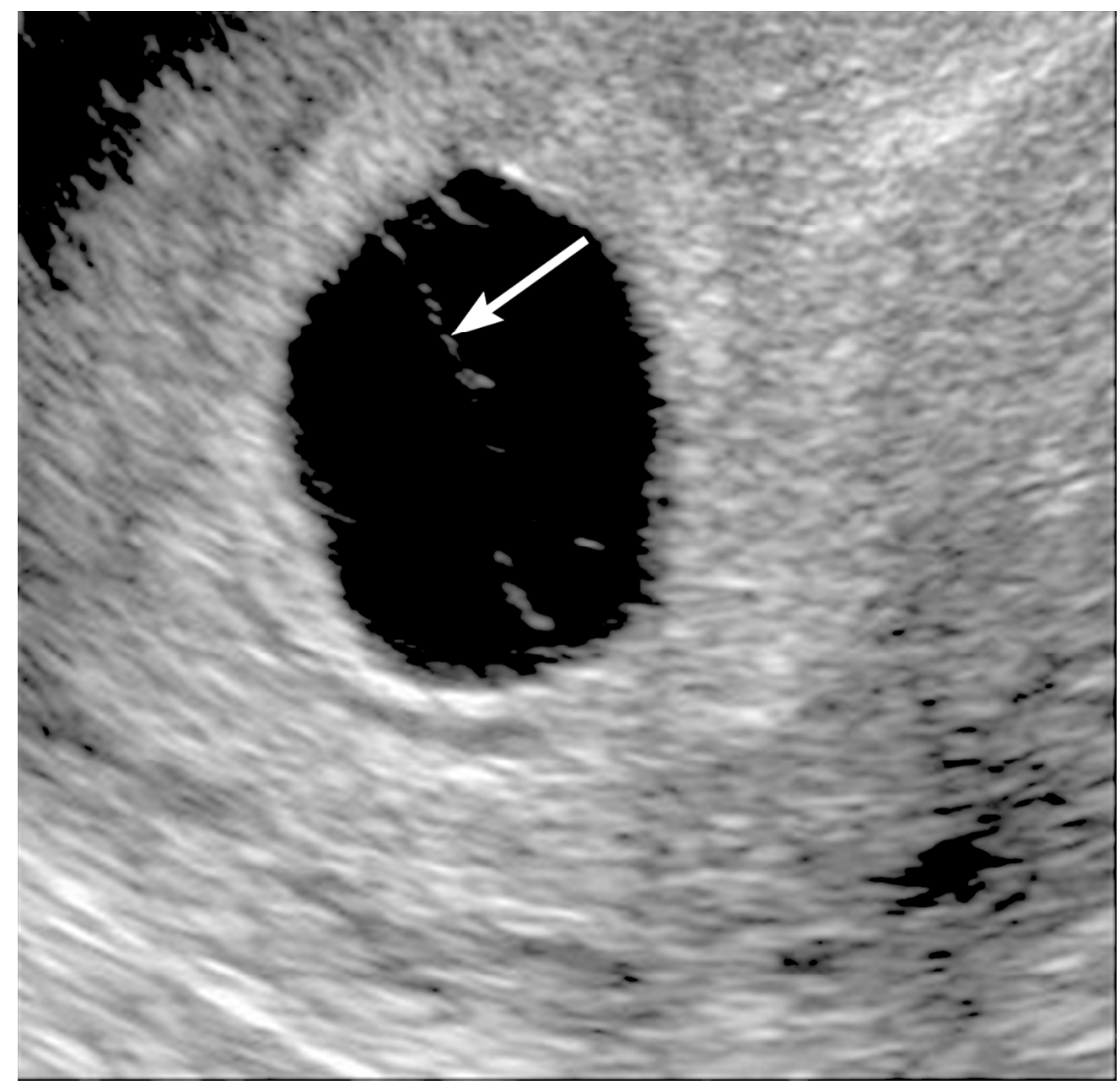

(Figure 3A) Figure 3: Septa in gestational sac illustrated in two women with molar pregnancies. A) Fine septa (arrow) within the gestational sac in a 37 year old woman with partial mole.

$127 \times 123 \mathrm{~mm}(300 \times 300 \mathrm{DPI})$

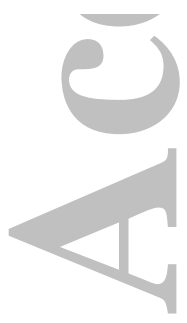




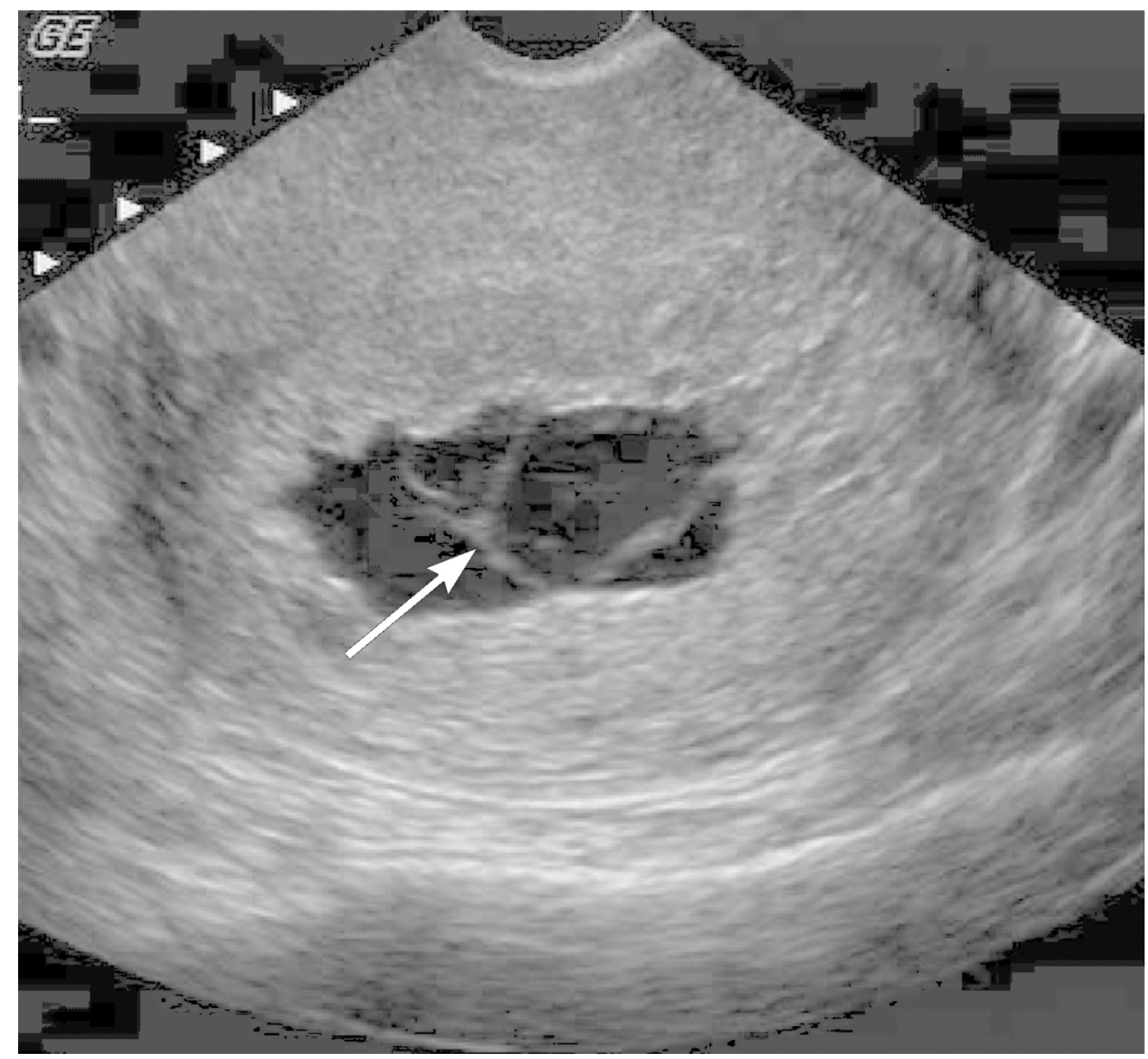

(Figure 3B) Figure 3: Septa in gestational sac illustrated in two women with molar pregnancies. B) Slightly thicker septa (arrow) within the gestational sac in a 23 year old woman with partial mole.

$127 \times 117 \mathrm{~mm}(300 \times 300$ DPI)

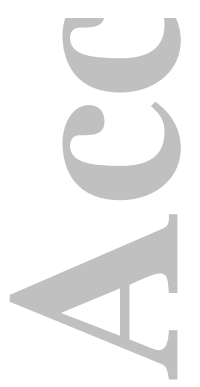

